\title{
Juvenile myasthenia gravis
}

\author{
Jyotiranjan Champatiray*, Anil Kumar Mohanty ${ }^{\#}$, Pradeep Sivaraj $\$$, \\ Jyotiranjan Satapathy ${ }^{\$}$, Rakesh Kumar Routray ${ }^{\$}$ \\ *Assciate Professor, " Professor, ${ }^{\$}$ Resident, Dept of Paediatrics, SVPPGIP, Sishu Bhawan, SCB medical \\ College, Cuttack, Odisha- 753001, INDIA
}

\section{Introduction:}

Juvenile myasthenia gravis is a rare disorder acquired in childhood, representing $10 \%$ to $15 \%$ of all cases of myasthenia gravis ${ }^{1}$. It is generally characterized by an autoimmune attack on acetylcholine receptors at the neuromuscular junction. Here, we report a patient with juvenile myasthenia gravis, presented to us with ptosis and drooling of saliva.

\section{Case description}

A 5 year old female child presented with cough, cold, fast breathing and drooling of saliva. Child was born out of non consanguineous marriage, without any perinatal insult during delivery or developmental delay. No history of sibling death or autoimmune disorders in the family. On examination, there was ptosis (Figure 1) which was more significant during the late in the evening and other neurological and systemic examination was unremarkable. Ophthalmological examination was done revealed ptosis without any gaze evoked nystagmus. Routine blood investigations were within normal limits. With progressive ptosis towards the end of the day, Juvenile Myasthenia gravis was suspected. Acetyl cholinesterase (AcHE) test was done with subcutaneous injection of neostigmine which showed a dramatic response (Figure 2). Repetitive nerve stimulation RNS study showed significant decremental response $(>10 \%)$ in orbicularis oculi, Right and Left trapezius muscle, suggestive of post synaptic neuromuscular junction (NMJ) receptor disorder. Computerized tomography CT scan of thorax was done to rule out thymic hyperplasia. We diagnosed this child as a case of juvenile myasthenia gravis and treated with pyridostigmine. The child improved symptomatically and under the follow up (Figure 3).

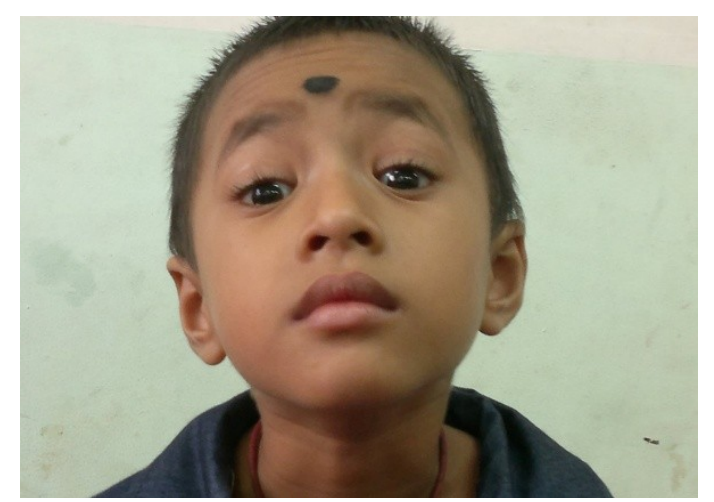

Figure 1: A 5 yr old girl with clinically evident ptosis and compensatory head elevation.

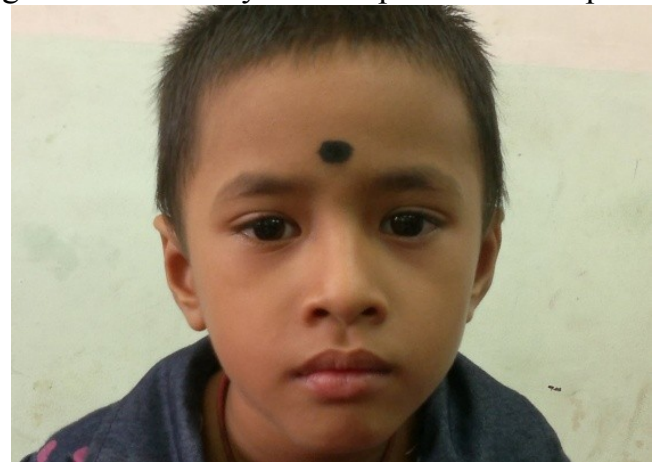

Figure 2: Same girl showed dramatic response to neostigmine test 


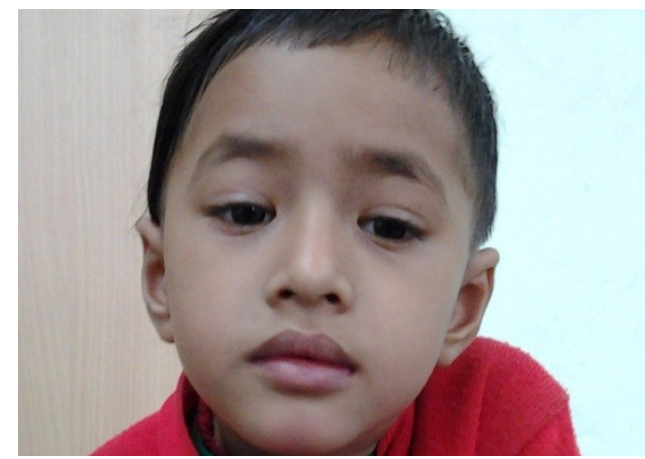

Figure 3: During follow up the child had reduced degree of ptosis.

\section{Discussion}

Myasthenia gravis (MG) in childhood and adolescence is rare comprising 10 to $20 \%$ of all myasthenic patients $^{2,3}$. Myasthenia gravis is a chronic disease characterized by rapid fatigability of striated muscle. There are 3 clinical forms of MG in children: juvenile myasthenia gravis, congenital myasthenia gravis, and transient myasthenia gravis. The most common cause is an autoimmune mediated blockade of acetyl choline receptors (AchR) with elevated anti AchR antibodies. Girls are more commonly affected than boys $(1.3-1.8)^{4,5}$. In Morita et al, Most of the cases presented between 9 to 12 years of age ${ }^{6}$. Our case was also a female child presented in the early age.

In Morita et al study most of the patients $94.4 \%$ had ptosis as one of the initial symptoms. Ptosis was bilateral in $88 \%$ patients and unilateral in $12 \%$. Other symptoms like diplopia, bulbar signs, bulbar and limb weakness were present in $61 \%, 50 \%, 55 \%, 66.6 \%$ cases. Similarly we also had the child with ptosis and drooling of saliva. The diagnosis is based essentially on the patient's history and clinical_examination with a positive anticholinesterase (edrophonium or neostigmine) test ${ }^{7}$. EMG with repetitive stimulation and single-fiber EMG are useful for the diagnosis ${ }^{3}$. Serum acetylcholine receptor antibodies (AchR Ab) are important to confirm the diagnosis but are more frequently negative when compared to adults. Antibody-negative myasthenia gravis were not rare in children ${ }^{3-5}$. We diagnosed this child based on clinical and neostigime test, but we could not do AchR auto antibodies level which was our limitation.

In morita et al study, patients were treated with oral doses of pyridostigmine bromide, 30 to $300 \mathrm{mg}$ per day. $12.5 \%$ had a complete remission in 1 to 3 months, $18.7 \%$ cases improved, $12.5 \%$ cases remained the same, and $56.2 \%$ worsened $^{6}$. Other treatment of myasthenic children includes immunosuppresents (mainly prednisone and azathioprine), plasmapheresis, intravenous high-dose immunoglobulins (IVIg), and thymectomy ${ }^{7}$. We treated the child with pyridostigmine, his ptosis and other symptoms resolved. The patient is under the follow up for 6 months without any of the symptoms.

\section{References}

[1]. Gadient $P^{l}$, Bolton J, Puri V. Juvenile myasthenia gravis: three case reports and a literature review. J Child Neurol. 2009 May;24(5):584-90. doi: 10.1177/0883073808325651.

[2]. Szorbor A, Máttyus A, Molnár J. Myasthenia gravis in childhood and adolescence: report on 209 patients and review of literature. Acta Paediatr Hung 1988-1989;29:299-312.

[3]. Snead OC, Benton JW, Dwyer D, et. al. Juvenile myasthenia gravis. Neurology 1980;30:732-739.

[4]. Andrew PI, Massey JM, Sanders DB. Acetylcholine receptor antibodies in juvenile myasthenia gravis. Neurology 1993;43:977-982.

[5]. Evoli A, Batocchi AP, Bartoccioni E, Lino MM, Minisci C, Tonali P. Juvenile myasthenia gravis with prepubertal onset. Neuromuscul Disord 1998;8:561-567.

[6]. 6. Maria da Penha A. Morita, Alberto A. Gabbai, Acary S.B. Oliveira, Audrey S. Penn. Myasthenia Gravis In Children Analysis of 18 patients Arq. Neuro-Psiquiatr. 2001; 59(3B): http://dx.doi.org/10.1590/S0004-282X2001000500005

[7]. Swaiman KF. Diseases of the neuromuscular junction. In Swaiman KF (ed.) Pediatric neurology. 2.Ed. Saint Louis: Mosby, 1994:1453-1476. 